\title{
MR Imaging of Hippocampal Asymmetry at 3T in a Multiethnic, Population-Based Sample: Results from the Dallas Heart Study
}

\author{
R.T. Lucarelli, R.M. Peshock, R. McColl, K. Hulsey, C. Ayers, A.R. Whittemore, and K.S. King
}

\begin{abstract}
BACKGROUND AND PURPOSE: Asymmetry of the hippocampus is regarded as an important clinical finding, but limited data on hippocampal asymmetry are available for the general population. Here we present hippocampal asymmetry data from the Dallas Heart Study determined by automated methods and its relationship to age, sex, and ethnicity.
\end{abstract}

MATERIALS AND METHODS: 3D magnetization-prepared rapid acquisition of gradient echo MR imaging was performed in 2082 DHS-2 participants. The MR images were analyzed by using 2 standard automated brain-segmentation programs, FSL-FIRST and FreeSurfer. Individuals with imaging errors, self-reported stroke, or major structural abnormalities were excluded. Statistical analyses were performed to determine the significance of the findings across age, sex, and ethnicity.

RESULTS: At the 90 th percentile, FSL-FIRST demonstrated hippocampal asymmetry of $9.8 \%$ ( $95 \% \mathrm{Cl}, 9.3 \%-10.5 \%)$. The 90 th percentile of hippocampal asymmetry, measured by the difference in right and left hippocampi volume and the larger hippocampus, was $17.9 \%$ (95\% $\mathrm{Cl}$, $17.0 \%-19.1 \%)$. Hippocampal asymmetry increases with age $(P=.0216)$, men have greater asymmetry than women as shown by FSL-FIRST $(P=$ .0036), but ethnicity is not significantly correlated with asymmetry. To confirm these findings, we used FreeSurfer. FreeSurfer showed asymmetry of $4.4 \%(95 \% \mathrm{Cl}, 4.3 \%-4.7 \%)$ normalized to total volume and $8.5 \%(95 \% \mathrm{Cl}, 8.3 \%-9.0 \%)$ normalized by difference/larger hippocampus. FreeSurfer also showed that hippocampal asymmetry increases with age $(P=.0024)$ and that men had greater asymmetry than women $(P=.03)$.

CONCLUSIONS: There is a significant degree of hippocampal asymmetry in the population. The data provided will aid in the research, diagnosis, and treatment of temporal lobe epilepsy and other neurologic disease.

ABBREVIATIONS: $\mathrm{Cl}$ = confidence interval; DHS = Dallas Heart Study; FIRST = FSL Integrated Registration and Segmentation Tool; MPRAGE = magnetizationprepared rapid acquisition of gradient echo

A symmetry of the hippocampus is relevant in neurologic disease and is an important clinical finding. However, we lack hippocampal asymmetry data representative of the general population to guide the interpretation of hippocampal asymmetry.

Received May 16, 2012; accepted after revision July 6.

From the Departments of Radiology (R.T.L., R.M.P., R.M., K.H., A.R.W., K.S.K.), and Internal Medicine (R.M.P.), and Donald W. Reynolds Cardiovascular Clinical Research Center (R.M.P., C.A.), University of Texas Southwestern Medical Center, Dallas, Texas; and Division of Cardiology (R.M.P., C.A.), Johns Hopkins, Baltimore, Maryland.

Grant support for the Dallas Heart Study was provided by the Donald W. Reynolds Foundation and by US Public Health Service General Clinical Research Center grant M01-RR00633 from National Institutes of Health (NIH)/National Center for Research Resources, Clinical Research (NCRR-CR). R.T.L. was supported by the Doris Duke Charitable Foundation Clinical Research Fellowship. K.S.K. was supported by grants KL2RR024983, and ULIRR024982 titled North and Central Texas Clinical and Translational Science Initiative (Robert Toto, MD, Principal Investigator) from the NCRR, a component of the NIH, and NIH Roadmap for Medical Research. Information on NCRR is available at http://www.ncrr.nih.gov/. Information on re-engineering the clinical research enterprise can be obtained from

http://nihroadmap.nih.gov/clinicalresearch/overview-translational.asp
Additionally, the reliability of prior work analyzing asymmetry by using user-guided segmentation has recently been called into question. ${ }^{1}$ At present, to our knowledge, there are no large studies with broad ethnic representation using high-resolution MR imaging, with consistent application of automated measures of the hippocampus to define the range of values of hippocampal asymmetry in the general population.

Hippocampal asymmetry is one of the most important findings suggestive of mesial temporal sclerosis, the most commonly

The views herein are solely the responsibility of the authors and do not necessarily represent the official views of the NCRR or $\mathrm{NIH}$.

Paper previously presented in part at: 50th Annual Meeting of the ASNR and the Foundation of the ASNR Symposium; April 21-26, 2012; New York New York.

Please address correspondence to Kevin S. King, MD, Department of Radiology, University of Texas Southwestern Medical Center, 5323 Harry Hines Blvd, Dallas, TX 75390-8896; e-mail: Kevin.King@UTSouthwestern.edu

- Indicates open access to non-subscribers at www.ajnr.org

三 Indicates article with supplemental on-line tables

http://dx.doi.org/10.3174/ajnr.A3308 
diagnosed structural cause of temporal lobe epilepsy, ${ }^{2}$ and is used to lateralize the seizure focus for epilepsy surgery. ${ }^{3-7}$ Other imaging findings in the hippocampus of mesial temporal sclerosis include T2 prolongation on MR imaging and loss of internal architecture. ${ }^{8}$ Hippocampal asymmetry is associated with mild cognitive impairment and Alzheimer disease, providing a potential biomarker for early diagnosis that has been reported to be more accurate than bilateral hippocampal atrophy. ${ }^{9}$ Additionally, several studies have suggested a link between asymmetry of the hippocampus and depression and schizophrenia. ${ }^{10-12}$

We report a study of a large population-based probability sample with broad ethnic and age representation to ascertain the distribution of hippocampal asymmetry and its variance in relation to age, ethnicity, and sex.

\section{MATERIALS AND METHODS \\ Study Population}

The Dallas Heart Study is a large multiethnic population-based cohort study of Dallas County residents that began in 1999. The DHS study was designed to produce unbiased population estimates of biologic and social variables, as has been previously described. ${ }^{13}$ Briefly, population sampling was based on US Postal Service delivery sequence files with selection probabilities increased for strata with larger concentrations of African Americans so that they would constitute approximately half the study sample. The study was approved by the institutional review board at the University of Texas Southwestern Medical Center, and all participants provided written informed consent.

Between September 2007 and December 2009, original DHS subjects were asked to participate in a continuation of the original study termed the Dallas Heart Study-2. Family members and spouses of the original participants were able to participate in the DHS-2. Participants underwent MR imaging at the University of Texas Southwestern Medical Center. Individuals with previous surgery for an aneurysm in the brain; metal fragments in the eyes, brain, or spinal canal; cardiac pacemaker; implantable cardiodefibrillators; cochlear implant; spinal cord stimulators or other internal electrical devices; pregnancy; and occupations associated with exposure to metal fragments were excluded from MR imaging.

A total of 2082 participants underwent brain MR imaging. Thirty-seven were excluded for self-reported stroke. Images of outliers as found by Robust Minimum Covariance Distance analysis of brain segments, ${ }^{14,15}$ individuals flagged for exclusion in previous DHS2 MR imaging brain studies, and individuals who had error flags generated during automated analysis were reviewed by a neuroradiologist (K.S.K.). On MR imaging review, 70 individuals with major structural defects (such as corpus callosum agenesis, imaging evidence of stroke, and hydrocephalus) or image-acquisition errors (such as metal and motion artifacts and other noise) were excluded. In total, 107 individuals were excluded from subsequent analysis.

\section{MR Imaging Protocol}

Brain MR images were obtained on a 3T MR imaging scanner (Achieva; Philips Healthcare, Best, the Netherlands) by using
3D magnetization-prepared rapid acquisition of gradient echo. Images were obtained from the vertex of the skull to the foramen magnum in true axial orientation. Specifications for 3D MPRAGE were the following: axial sections reconstructed at 1.0-mm section thickness; TR, $9.6 \mathrm{~ms}$; TE, $5.8 \mathrm{~ms}$; flip angle, $12^{\circ}$; FOV, $260 \times 260$ $\mathrm{mm}$ with a voxel size of $1.0 \times 0.9 \times 0.9 \mathrm{~mm}$.

\section{Image Analysis}

MR imaging quantification was performed by using the freely available FMRIB Software Library, FSL-FIRST (http://www.fmrib. ox.ac.uk/fsl/first/index.html). ${ }^{16,17}$ Volumes of the left and right hippocampi were derived from 3D MPRAGE sequences. In brief, the skull was removed from the 3D MPRAGE images, and the remaining images were segmented into 3 classes: CSF, WM, and gray matter. A mask for the hippocampus was created by using the FSL-Toolkit. Volumetric data were collected by using the FSLstats routine (http://www.fmrib.ox.ac.uk/fsl/avwutils/index.html). The present study focused on the hippocampus; therefore, the cerebellum and brain stem were excluded from analysis.

Further MR imaging quantification was performed by using the FreeSurfer image analysis suite, Version 4.4, which is documented and freely available for download on-line (http://surfer. nmr.mgh.harvard.edu/). The fully automated analysis was run at the Texas Advanced Computing Center at The University of Texas at Austin. Volumes of the left and right hippocampi, along with other cortical and subcortical structures not reported here, were derived from MPRAGE sequences. Individuals who had a Talairach atlas registration error $(n=11)$ had the atlas manually aligned following the procedures in the FreeSurfer documentation, and the images were reanalyzed. Images of individuals with minor errors on analysis $(n=2)$ or timeout errors $(n=9)$ were reanalyzed, and the masks generated by FreeSurfer were verified by a neuroradiologist (K.S.K.).

Hippocampal asymmetry was calculated by taking the absolute value of the difference of left and right hippocampal volume and dividing by the total hippocampal volume. Another measure that has practical significance when interpreting MR imaging was calculated by taking the value of the difference between the left and right volume and dividing by the larger of the right or left hippocampus.

\section{Statistical Analysis}

Statistical analyses were performed by using SAS, Version 9.2.0 (SAS Institute, Cary, North Carolina). Ninety-five percent distribution-free confidence intervals were generated with the method of Hahn and Meeker. ${ }^{18}$ Differences in hippocampal asymmetry as measured by FSL-FIRST for sex were evaluated with the 2-sided Mann-Whitney $U$ test at a significance level of $<.05$. Differences in hippocampal asymmetry as measured by FreeSurfer, to confirm FSL-FIRST results, were evaluated with a 1-sided MannWhitney $U$ test at a significance level of $<.05$. The correlation between age and hippocampal asymmetry was evaluated by using a Spearman rank-order correlation at a significance level of $<.05$. Mean age among men and women was compared by using the Student $t$ test, and among whites, Hispanics, and African Americans, by using ANOVA with the Tukey multiple comparison procedure. We evaluated the correlation between ethnicity and hip- 
Table 1: FSL-FIRST and FreeSurfer measures of hippocampal asymmetry in the total population and grouped by sex ${ }^{\mathrm{a}}$

\begin{tabular}{lcccc}
\hline \multicolumn{5}{c}{ Hippocampal Asymmetry } \\
\hline & 25th Percentile & 50th Percentile & 75th Percentile & 90th Percentile \\
\hline FSL-FIRST & & & & \\
$\quad$ Total & $1.6 \%(1.5 \%-1.7 \%)$ & $3.6 \%(3.4 \%-3.8 \%)$ & $6.2 \%(5.9 \%-6.6 \%)$ & $9.8 \%(9.3 \%-10.54 \%)$ \\
Men & $1.8 \%(1.5 \%-1.9 \%)$ & $3.8 \%(3.5 \%-4.2 \%)$ & $6.8 \%(6.2 \%-7.3 \%)$ & $11.2 \%(9.9 \%-11.8 \%)$ \\
$\quad$ Women & $1.5 \%(1.4 \%-1.7 \%)$ & $3.4 \%(3.1 \%-3.7 \%)$ & $5.9 \%(5.7 \%-6.3 \%)$ & $9.2 \%(8.7 \%-9.9 \%)$ \\
FreeSurfer & & & & \\
$\quad$ Total & $0.8 \%(0.7 \%-0.8 \%)$ & $1.7 \%(1.6 \%-1.8 \%)$ & $2.9 \%(2.8 \%-3.1 \%)$ & $4.5 \%(4.3 \%-4.7 \%)$ \\
Men & $0.8 \%(0.7 \%-0.9 \%)$ & $1.8 \%(1.6 \%-1.9 \%)$ & $3.2 \%(2.9 \%-3.3 \%)$ & $4.7 \%(4.4 \%-5.0 \%)$ \\
Women & $0.8 \%(0.7 \%-0.8 \%)$ & $1.7 \%(1.6 \%-1.8 \%)$ & $2.9 \%(2.8 \%-3.1 \%)$ & $4.5 \%(4.3 \%-4.7 \%)$ \\
\hline
\end{tabular}

${ }^{a}$ Asymmetry is defined by the absolute difference in left to right hippocampal volumes with respect to total hippocampal volume. Numbers in parentheses are $95 \%$ Cls.

Table 2: FSL-FIRST measures of hippocampal asymmetry ${ }^{a}$

\begin{tabular}{llccc}
\hline \multicolumn{5}{c}{ Hippocampal Asymmetry } \\
\hline & 25th Percentile & 50th Percentile & 75th Percentile & 90th Percentile \\
\hline FSL-FIRST & $3.1 \%(2.9 \%-3.4 \%)$ & $6.9 \%(6.5 \%-7.2 \%)$ & $11.7 \%(11.3 \%-12.4 \%)$ & $17.9 \%(17.0 \%-19.1 \%)$ \\
FreeSurfer & $1.5 \%(1.4 \%-1.6 \%)$ & $3.3 \%(3.1 \%-3.5 \%)$ & $5.7 \%(5.4 \%-5.9 \%)$ & $8.5 \%(8.3 \%-9 \%)$ \\
\hline
\end{tabular}

${ }^{a}$ Asymmetry defined by the absolute difference in left to right hippocampal volumes with respect to the volume of the larger side. Numbers in parentheses are $95 \%$ Cls.

pocampal asymmetry, adjusting for age as a covariate by using the general nonparametric approach developed by Schacht et al. ${ }^{19}$

\section{RESULTS}

After exclusion analysis, 1975 individuals were evaluated with a sex makeup of $58.3 \%(n=1151)$ female, and $41.7 \%(n=824)$ male and an ethnic makeup of $46.3 \%(n=914)$ African American, $37.2 \%(n=735)$ white, $14.1 \%(n=279)$ Hispanic, and $2.4 \%$ $(n=47)$ other or not reported. The mean age was $49.8 \pm 10.5$ years.

The degree of hippocampal asymmetry and its relationship to sex is shown in Tables 1 and 2. FSL-FIRST demonstrated significant asymmetry in our population (Table 1). After we normalized the difference in left and right hippocampal volume compared with the total hippocampal volume, the magnitude of hippocampal asymmetry at the 90th percentile for FSL-FIRST was $9.8 \%$ (95\% CI, 9.3\%-10.5\%). When one clinically evaluates images for asymmetry, the magnitude is typically assessed by comparing the size of the smaller hippocampus with that of the larger, and this measure is shown in On-line Table 1. FSL-FIRST also showed that men had significantly more asymmetry than women $(P=.0036)$. The median degree of hippocampal asymmetry for men was $3.8 \%$ (95\% CI, 3.5\%-4.2\%) and 3.4\% (95\% CI, 3.1\%-3.7\%) for women. The magnitude of asymmetry was also shown to increase with age $(P=.0216)$ when evaluated with FSL-FIRST, and it increased from $2.7 \%$ in the second decade to $4.5 \%$ in the seventh decade (Fig 1). This increase in hippocampal asymmetry with age was more pronounced in the top 90th percentile of individuals and increased from $9.9 \%$ to $11.3 \%$ as measured by FSL-FIRST (Fig 1). When evaluating ethnic differences in hippocampal asymmetry, we found that there was a significant difference in age among Hispanics, whites, and African Americans $(P<.0001)$. After controlling for differences in age, we found no significant correlation between ethnicity and hippocampal asymmetry.

To confirm the findings with FSL-FIRST, another automated program, FreeSurfer, was used to evaluate asymmetry as shown in Tables 1 and 2. FreeSurfer also showed a significant degree of asymmetry in our population when we normalized asymmetry by taking the difference between the left and right hippocampi and the total hippocampal volume (Table 1). This difference in asymmetry was also apparent when we normalized the difference between the left and right hippocampi and the larger of the 2 hippocampi (Table 2). FreeSurfer further demonstrated an increase in hippocampal asymmetry with increasing age $(P=.0024)$ as shown in Fig 1. The difference between men and women seen with FSL-FIRST was confirmed with FreeSurfer $(P=.03)$, but ethnicity was not associated with asymmetry when controlled for age.

\section{DISCUSSION}

Our study reports a detailed description of the magnitude of hippocampal asymmetry analyzed with fully automated segmentation methods by using high-magnetic-field-strength MR imaging among participants in the Dallas Heart Study. To our knowledge, this is the first description of the magnitude of hippocampal asymmetry with evaluation of distributions by age, sex, and ethnicity in a multiethnic population-based probability sample of community-dwelling individuals. We initially performed our analysis by using FSL-FIRST and noted a significant degree of hippocampal asymmetry in the population. To confirm these findings, we used a second commonly used software package, FreeSurfer, which confirmed the findings of hippocampal asymmetry in our population. We took a conservative approach by reporting quartiles and the top 90th percentile; yet by definition, $10 \%$ of individuals in our study have an even greater degree of asymmetry than reported here (more detailed results are shown in On-line Tables 1 and 2). We chose to report asymmetry in 2 different ways. Most of our analysis used a traditional approach, reporting hippocampal asymmetry normalized by total hippocampal volume (Table 1 and Fig 1). However, we also reported hippocampal asymmetry normalized by the larger of the 2 hippocampi because this reflects the evaluation when attempting to visually determine symmetry between hippocampi in a clinical setting (Table 2).

This work expands our knowledge of hippocampal asymmetry in several ways. Our current conceptions regarding hippocampal asymmetry are based largely on studies with user-guided segmentation performed by experts in neuroanatomy. However, a recent analysis of user-guided segmentation has shown the technique to 
be flawed. By presenting randomly inserted mirror images for analysis, investigators were able to determine a consistent left-right bias in subcortical segmentation, which was most significant for the hippocampus, in some cases as high as $11 \%$. The authors proposed "laterality of visual perception" as an inherent limitation in any study using user interaction in hippocampal segmentation and further suggested a reappraisal of prior work in light of this finding. ${ }^{1}$ Thus, data previously reported by using manual segmentation have the potential for material bias.

Previous studies of hippocampal volumes and asymmetry were limited by the time-intensive nature of userguided segmentation, and as a result, no large population-based studies have been conducted. Pedraza et $\mathrm{al}^{20}$ sought to address the small size of these previous studies by performing a meta-analysis on 82 studies for a total of 3564 participants from the control groups. This approach primarily addresses type II errors or low power among prior studies. This approach does not, however, address selection bias because control groups are not designed to reflect the general population. ${ }^{21,22}$ Using automated techniques, we were able to examine data from 1975 individuals by using the same segmentation methods, imaging techniques, and MR imaging hardware.

Because signal strength may influence image quality and thus the accuracy of segmentation, our analysis was conducted by using 3T 3D-MPRAGE images reconstructed at 1-mm sections, providing high-resolution volumetric images compared with previous $1.5 \mathrm{~T}$ studies. The amount of hippocampal asymmetry demonstrated is higher than that reported in previous studies, and while the reason for this is presently unknown and may be due to technical improvements in our study, it could also be due to other existing pathology present in a population-based sample that may affect the hippocampal asymmetry seen in our study participants.

Prior studies have validated the automated methods used for the segmentation of deep brain nuclei by using manual segmentation and postmortem examination as the criterion standard references. ${ }^{23-25}$ While the validity of manual segmentation itself has recently been called into question, ${ }^{1}$ one would not expect these automated measures to have the same systematic bias as manual tracing. Recently there has been considerable effort to compare the sensitivity of these automated methods in determining hippocampal volume. Morey et $\mathrm{al}^{26}$ and Pardoe et $\mathrm{al}^{27}$ in separate
FSL-FIRST: Hippocampal Asymmetry Increases With Age

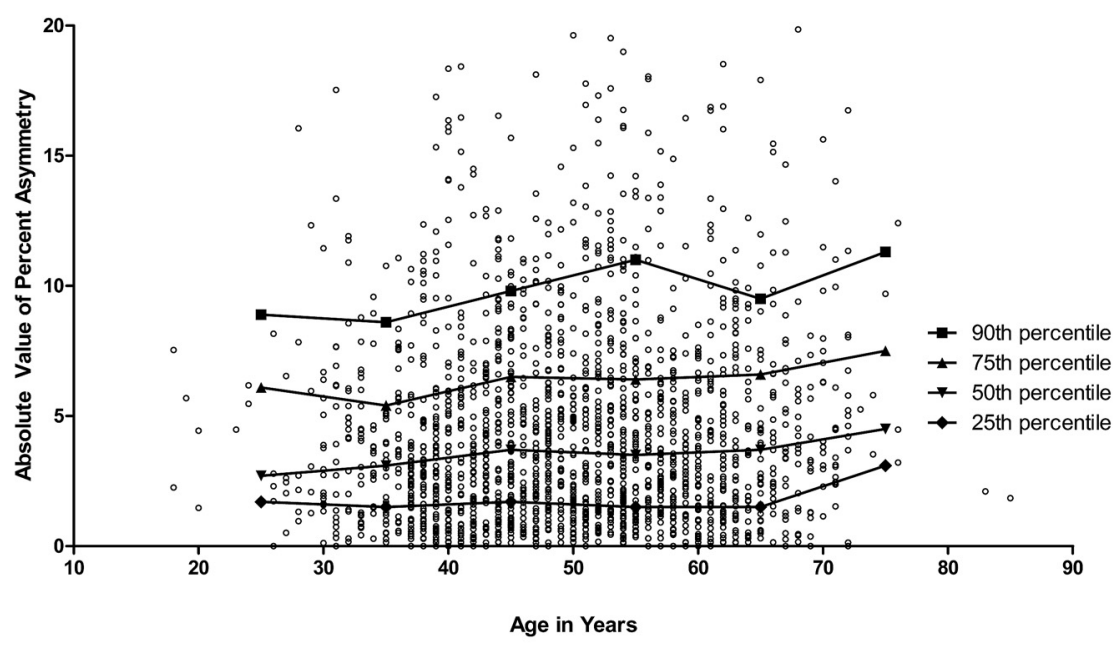

Freesurfer: Hippocampal Asymmetry Increases With Age

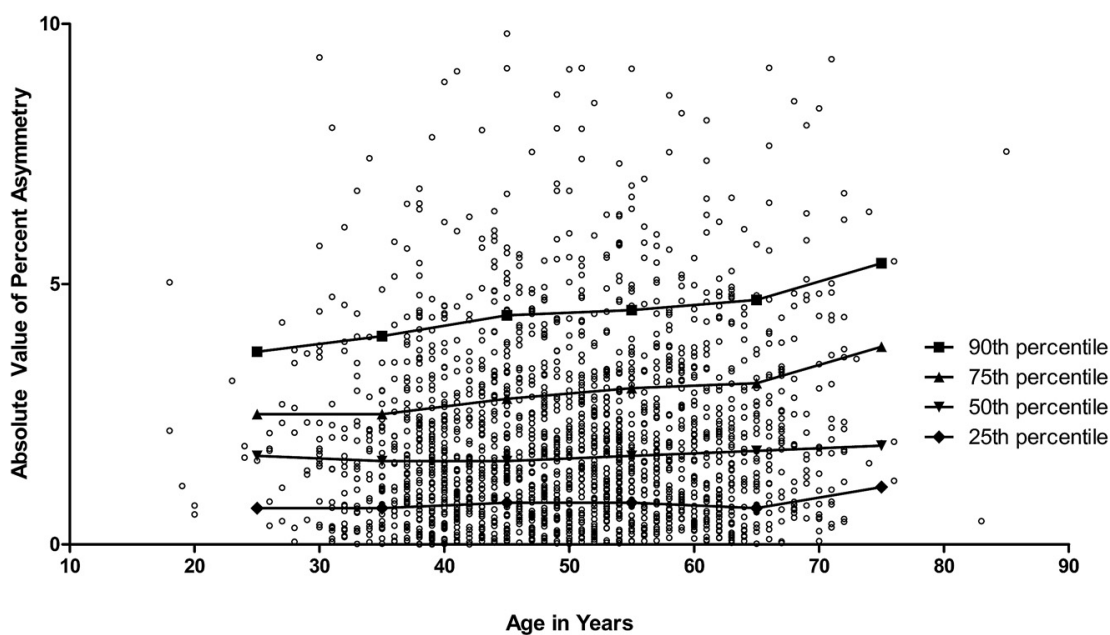

Age in Years

FIG 1. Hippocampal asymmetry increases with age as measured by FSL-FIRST (top) and FreeSurfer (bottom); ( $<1 \%$ of points were not plotted to preserve scale): second decade, $n=28$; third decade, $n=331$; fourth decade, $n=613$; fifth decade, $n=601$; sixth decade, $n=344$; seventh decade, $n=53$; 1st and 8th decades not shown, $n=5$.

studies both concluded that FreeSurfer is more sensitive at detecting hippocampal atrophy than FSL-FIRST. Pardoe et al further concluded that FreeSurfer is less likely than FSL-FIRST to fail to detect atrophy of the hippocampus compared with manual segmentation. ${ }^{27}$ Notably, FSL-FIRST was found to have greater variation than FreeSurfer when determining hippocampal volume compared with manual tracing. ${ }^{26}$ However, FSL-FIRST has several advantages compared with FreeSurfer, including much faster processing time and requiring considerably less computing resources. Such prior validation efforts and our own analysis are designed for accurate assessment of hippocampal asymmetry in the population and targeted groups. This work does not advocate replacing qualitative analysis of MR images for individual clinical diagnosis. Additionally, while our data show a significant degree of hippocampal asymmetry in the general population as measured by automated methods, it is not clear at what magnitude this asymmetry would be detected clinically. It would be fruitful to investigate what percentage differ- 
ence in hippocampal asymmetry is detectable clinically by expert reviewers, to further elucidate the impact of the findings reported herein.

The sample we have evaluated in this study was designed to be representative of the adult population of Dallas County, with oversampling to ensure that approximately half our population was African American. We evaluated hippocampal asymmetry with respect to ethnicity and did not see a significant difference in our sample. We did not evaluate or exclude any persons on the basis of their neurologic or psychiatric histories. Our study is designed to be reflective of the general population without a history of stroke. Diseases that have a high prevalence in the general population that do not preclude independent living in the community are likely included in this sample. In the case of epilepsy, prior studies of prevalence would suggest we would have approximately 5 individuals per 1000 with a history of epilepsy. ${ }^{28}$ Because we focused on median values and percentile ranges and used nonparametric analysis, the effect of specific outliers is limited. However, early changes that may lead to mild cognitive impairment or eventually Alzheimer disease may be observed in our study to the same extent that they would be observed among the general population.

Our data suggest a large degree of asymmetry in the general population, and they question how much weight should be placed on mild hippocampal asymmetry as a solitary imaging finding. For the example of mesial temporal sclerosis, we would suggest that future quantitative studies evaluate the degree of hippocampal asymmetry rather than presence or absence. Further studies should also include other abnormalities such as increased signal on FLAIR or loss of the normal internal architecture in a comprehensive predictive model. In the example of suspected mesial temporal sclerosis, a more comprehensive model may lead to improved diagnostic accuracy of presurgical imaging and more precise patient selection for epilepsy surgery.

\section{CONCLUSIONS}

The data presented herein on the magnitude of hippocampal asymmetry provide the first large population-based study with broad ethnic representation by using high-resolution MR imaging and fully automated methods. Our findings suggest that age and sex should be considered when evaluating hippocampal asymmetry and that caution is warranted in the interpretation of hippocampal asymmetry as an indicator of pathology.

\section{ACKNOWLEDGMENTS}

The authors acknowledge Myron Weiner, MD, for his contributions to this project. We further appreciate the statistical support provided by Linda Hynan, PhD. We acknowledge the Texas Advanced Computing Center at the University of Texas at Austin for providing high-performance computing resources that have contributed to the research results reported within this article (http://www.tacc.utexas.edu). We thank the staff of the University of Texas Southwestern and the Dallas Heart Study for their important contributions.
Disclosures: Richard T. Lucarelli-RELATED: Support for Travel to Meetings for the Study or Other Purposes: Doris Duke Charitable Foundation Clinical Research Fellowship, Other: Doris Duke Charitable Foundation Clinical Research Fellowship, Comments: Supported by a stipend from the Doris Duke Charitable Foundation Clinical Research Fellowship. Ronald M. Peshock—RELATED: Grant: Reynolds Foundation, * Support for Travel to Meetings for the Study or Other Purposes: Reynolds Foundation.* Kevin S. King-RELATED: Grant: NIH CTSA grant,* Comments: NIH Funding: 5 KL2 RR024983-0520, Principal Investigator: Robert Toto, MD; K12 mentored career award program at the University of Texas Southwestern Medical School at Dallas, Role: Clinical Scholar Award to promote development as an independent investigator. *Money paid to the institution.

\section{REFERENCES}

1. Maltbie E, Bhatt K, Paniagua B, et al. Asymmetric bias in user guided segmentations of brain structures. NeuroImage 2012;59:1315-23

2. Blümcke I. Neuropathology of focal epilepsies: a critical review. Epilepsy Behav 2009;15:34-39

3. Breier JI, Leonard CM, Bauer RM, et al. Quantified volumes of temporal lobe structures in patients with epilepsy. J Neuroimaging 1996;6:108-14

4. Gilmore RL, Childress MD, Leonard C, et al. Hippocampal volumetrics differentiate patients with temporal lobe epilepsy and extratemporal lobe epilepsy. Arch Neurol 1995;52:819-24

5. Radhakrishnan K, So EL, Silbert PL, et al. Predictors of outcome of anterior temporal lobectomy for intractable epilepsy: a multivariate study. Neurology 1998;51:465-71

6. Salanova V, Markand O, Worth R. Longitudinal follow-up in $\mathbf{1 4 5}$ patients with medically refractory temporal lobe epilepsy treated surgically between 1984 and 1995. Epilepsia 1999;40:1417-23

7. Watson C, Jack CR Jr, Cendes F. Volumetric magnetic resonance imaging: clinical applications and contributions to the understanding of temporal lobe epilepsy. Arch Neurol 1997;54:1521-31

8. Woermann FG, Vollmar C. Clinical MRI in children and adults with focal epilepsy: a critical review. Epilepsy Behav 2009;15:40-49

9. Shi F, Liu B, Zhou Y, et al. Hippocampal volume and asymmetry in mild cognitive impairment and Alzheimer's disease: meta-analyses of MRI studies. Hippocampus 2009;19:1055-64

10. Kronmüller KT, Schroder J, Kohler S, et al. Hippocampal volume in first episode and recurrent depression. Psychiatry Res 2009;174:62-66

11. Qiu A, Wang L, Younes L, et al. Neuroanatomical asymmetry patterns in individuals with schizophrenia and their non-psychotic siblings. Neuroimage 2009;47:1221-29

12. Xia J, Chen J, Zhou Y, et al. Volumetric MRI analysis of the amygdala and hippocampus in subjects with major depression. J Huazhong Univ Sci Technolog Med Sci 2004;24:500-02, 506

13. Victor RG, Haley RW, Willett DL, et al. The Dallas Heart Study: a population-based probability sample for the multidisciplinary study of ethnic differences in cardiovascular health. Am J Cardiol 2004;93:1473-80

14. Jackson DA, Chen Y. Robust principal component analysis and outlier detection with ecological data. Envirometrics 2004;15:129-39

15. Filzmoser P, Garrett RG, Reimann C. Multivariate outlier detection in exploration geochemistry. Computers \& Geosciences 2005;31:579-87

16. Smith SM, Jenkinson M, Woolrich MW, et al. Advances in functional and structural MR image analysis and implementation as FSL. Neuroimage 2004;2(suppl 1):S208-19

17. Woolrich MW, Jbabdi S, Patenaude B, et al. Bayesian analysis of neuroimaging data in FSL. Neuroimage 2009;45:S173-86

18. Hahn GJ, Meeker WQ. Statistical Intervals: A Guide for Practitioners. New York: John Wiley \& Sons; 1991

19. Schacht A, Bogaerts K, Bluhmki E, et al. A new nonparametric approach for baseline covariate adjustment for two-group comparative studies. Biometrics 2008;64:1110-16

20. Pedraza O, Bowers D, Gilmore R. Asymmetry of the hippocampus and amygdala in MRI volumetric measurements of normal adults. J Int Neuropsychol Soc 2004;10:664-78 
21. Kleinbaum DG, Morgenstern H, Kupper LL. Selection bias in epidemiologic studies. Am J Epidemiol 1981;113:452-63

22. Wacholder S, Silverman DT, McLaughlin JK, et al. Selection of controls in case-control studies. III. Design options. Am J Epidemiol 1992;135:1042-50

23. Fischl B, Salat DH, Busa E, et al. Whole brain segmentation: automated labeling of neuroanatomical structures in the human brain. Neuron 2002;33:341-55

24. Patenaude B, Smith SM, Kennedy DN, et al. A Bayesian model of shape and appearance for subcortical brain segmentation. Neuroimage 2011;56:907-22
25. Rosas HD, Liu AK, Hersch S, et al. Regional and progressive thinning of the cortical ribbon in Huntington's disease. Neurology 2002;58:695-701

26. Morey RA, Petty CM, Xu Y, et al. A comparison of automated segmentation and manual tracing for quantifying hippocampal and amygdala volumes. Neuroimage 2009;45:855-66

27. Pardoe HR, Pell GS, Abbott DF, et al. Hippocampal volume assessment in temporal lobe epilepsy: how good is automated segmentation? Epilepsia 2009;50:2586-92

28. Forsgren L, Beghi E, Oun A, et al. The epidemiology of epilepsy in Europe: a systematic review. Eur J Neurol 2005;12:245-53 